\title{
Mild Dehydration - Possible Association with Bladder and Colorectal Cancers - A Review
}

Daniel Benharroch* and Samuel Ariad

Department of Pathology and Oncology, Soroka University Medical Center and Faculty of Health Sciences, Ben-Gurion University of the Negev, Beer-Sheva, Israel

\begin{abstract}
Background: The role of water consumption/dehydration in cancer has not been fully clarified to date.

Objective: Review the role of water in bladder and colorectal cancers.

Methods: A critical review of the literature was performed, including urinary tract infection and constipation together with bladder and colorectal cancers relation to water ingestion.

Results: Numerous inconsistencies were found and their causes include, among others, the incapacity to adequately measure water intake, the lack of matching of low fluid intake with mild dehydration, the multifactorial nature of the evaluated diseases, and changing methods of investigation.

Conclusions: Notwithstanding the technical and clinical limitations, there is some support for the contribution of dehydration to the pathogenesis of the diseases described.
\end{abstract}

Keywords: Mild dehydration; Urinary tract infections; Bladder cancer; Constipation; Colorectal cancer

\section{Introduction}

Water is the core nutrient of life, though it is often forgotten in the inventory of dietary constituents. Daily hydration is generally well sustained, assuming that food and fluid are accessible, but no water reserves are available. Total water intake includes drinking water, and water in beverages and food. Deficits are unlikely, with requirements of $3.71 /$ day of water for adult men and 2.7 for adult women. Urine output is about $1-21 / d$, and is the main mechanism of water regulation, but output may vary widely [1].

There are major gaps in the knowledge related to the measurement of total fluid intake and hydration status at the population level. There are no available methods for assessing hydration status. Urine indices reflect the recent volume of fluid absorbed but not hydration [2].

Healthy humans regulate daily water balance in a precise manner, despite highly variable water needs and consumption and exposure to stressors of hydration status [1].

Risk factors for dehydration include reduced mobility, visual problems, confusion, medications (diuretics, laxatives, and sedatives), and all acute pathologies with fever, decreased dietary water intake and fear of incontinence. Thirst is a security mechanism, and does not regulate primary intake. This is particularly true in the elderly, in whom thirst should be considered a marker of the onset of dehydration. Dehydration risk factors in this age group include age $>85$, thirst reduction, difficult access to water, cognitive disorders, swallowing malfunction, reduced appetite and acute pathology [3]. They may also include occupational self-restriction and populations that have little or no access to drinking water. Elderly individuals will dehydrate more readily than younger adults since the sensation of thirst is reduced with aging, and with loss of autonomy or of cognitive functions, all of which limit access to water [3].

There is increasing and sometimes conflicting evidence that mild dehydration (excluding severe clinical dehydration) plays a role in various chronic diseases. However, mild dehydration is very difficult to evaluate objectively.
A relationship has been suggested between mild dehydration and several chronic diseases [4,5]. Urolithiasis is probably the disorder that is most convincingly linked with dehydration. Constipation, exercise asthma and hyperglycaemia in diabetic ketoacidosis are all associated with dehydration as demonstrated in quasi-experimental studies $[4,5]$. Urinary tract infections and cerebral infarction are associated with dehydration, as demonstrated in descriptive studies. Bladder and colon cancers are associated with dehydration, but the evidence is inconsistent $[4,5]$. The association of these chronic diseases with dehydration has not been firmly established. However, we live in a desert region, in the Negev highlands of Israel, and we have a tendency to believe that this relationship, considered by many as a myth, may nevertheless be genuine.

Evidence to date tends to support a role for low water intake in the development of UTI. A more precise evaluation of the eventual mild dehydration might possibly confirm its role in the pathogenesis of UTI $[4,5]$.

A significant association of bladder cancer with chronic UTI has been demonstrated [6]. The inverse relationship found by Wilkens et al. [7] between water intake and bladder cancer at a locale in which ground water was free of contaminants, supports the suggestion by Villanueva et al. [8] that as a universal solvent, water cannot be evaluated for its distinct fluid effect.

In contrast, a significant inverse association between the number

*Corresponding author: Daniel Benharroch, MD, Department of Pathology, Soroka University Medical Centre, P.O. Box 151, Beer-Sheva 84101, Israel, Fax: 792 8-6232770; E-mail: benaroch@bgu.ac.il

Received December 10, 2011; Accepted January 04, 2012; Published January 06, 2012

Citation: Benharroch D, Ariad S (2012) Mild Dehydration - Possible Association with Bladder and Colorectal Cancers - A Review. J Food Process Technol 3:142. doi:10.4172/2157-7110.1000142

Copyright: (c) 2012 Benharroch D, et al. This is an open-access article distributed under the terms of the Creative Commons Attribution License, which permits unrestricted use, distribution, and reproduction in any medium, provided the original author and source are credited. 
of glasses of tap water and reduced risk of colorectal cancer has been reported, especially in women [9].

The present review covers chronic diseases possibly associated with mild dehydration. Special emphasis was placed on urinary tract infection, bladder cancer, constipation and colorectal cancer, and their association with water and fluid intake and/or dehydration.

\section{Mild Dehydration and Urinary Tract Infections}

Urinary tract infections (UTIs) are frequent, with women developing at least one UTI in their lifetime. Based on empirical data and personal experience, clinicians recommend high fluid intake in order to prevent UTI, accompanied by frequent micturition, since water diuresis is believed to flush the urinary tract and frequent voiding is thought to prevent bacterial proliferation. Some may add prevention of constipation to the above [10].

On the other hand, animal studies suggest a protective effect for concentrated urine, but this may not be the case for humans. Clinical data on this issue are few and contradictory, perhaps because of the difficulty in evaluating water intake [10].

Mechanical factors like bladder emptying and the antibacterial activity of the bladder mucosa depend partially on urine output. Some reports on reduced fluid intake in patients with UTI describe even the "infrequent voiding syndrome" with an enlarged bladder that contracts poorly and demonstrates residual urine. Prophylaxis involves higher fluid intake to decrease urine osmolality and the incidence of UTI [10].

The above statements have been considered to be based on hearsay or myth and lack consistency, especially as clinical and animal studies are contradictory. Thus, further research using controlled prospective clinical studies is required to establish the harmful effect of dehydration on the urinary tract [10].

Studies by Stauffer [11] and Rudaitis [12] examined the risk factors for recurrent UTI. Poor fluid intake was observed in 53\% of patients with UTI (versus $16 \%$ in controls $-\mathrm{p}=0.001$ ) and infrequent voiding in $54 \%$ (versus $24 \%$ in controls $-\mathrm{p}=0.001$ ). A vesicoureteral reflux was found in a quarter of cases as well as in controls. Some of the girls followed-up urinated only once or twice daily, possibly due to cold and/or dirty toilet facilities. Bacterial proliferation was more likely due to the wider interval between voiding. This may lead to bladder dilatation and to partial bladder emptying.

The association between fluid consumption and UTI was also considered from an unusual point of view, examining the use of fluid intake by individuals as a means of reducing urinary symptoms. Patients with urinary frequency drank significantly more (13\% more) fluid ( $\mathrm{p}<0.001)$, while female patients with urgency drank significantly less fluid (9\% less) $(\mathrm{p}<0.047)[13]$.

\section{Fluid intake, Urinary Tract Infections and Bladder Cancer risk}

Of note, a possible shift between the effect of dehydration on UTIs and its effect on bladder cancer was reported by Jiang et al. [14]. Jiang et al found that female patients with a history of UTI that had occurred within 5 years or less showed a lower incidence of bladder cancer, but this finding did not reach statistical significance. Increased statistical significance was found in cases of recurrent UTI. In reports reviewed by Jiang and describing an association between UTI and bladder cancers, the findings were inconsistent, though overall, a trend for increased risk from UTI was observed. In rats, a positive link was found between chronic UTI and bladder cancer. An anticancer effect of antibiotics has been suggested and was supported by experimental evidence [14].

Kunze et al. [6] defined "bladder cancer" as all histological types of this neoplasm. When including mineral and tap water in total liquid intake, they found an increased risk of bladder cancer in individuals who drank 3 litres of fluid per day, as compared with 2 litres or less, $\mathrm{OR}=4.9$ [95\% CI: 2.0-12.3]. There was also a significant association of bladder cancer with chronic UTI, OR $=1.8$ [95\% CI: 1.2-2.7]. In contradiction with the "urogenous contact hypothesis" [15] (which suggest that concentrated urine with infrequent micturition, expose the bladder urothelium to carcinogens found in the urine), Kunze et al. [6] found a significant relation between higher fluid consumption, more frequent micturition and a reduction in the time of contact between urine and the urothelium and urothelial exposure to carcinogens. They note that urine concentration is lower in women and that men have a four-fold higher rate of occurrence of bladder cancer [6].

\section{Dehydration and Indirect Association with Bladder Cancer}

Numerous epidemiological studies, both selected case-control and cohort investigations, have been carried out since the 1960s, but the association of fluid intake with the risk of bladder cancer remains inconsistent.

An association between increased fluid intake accompanied with frequent micturition, and reduced risk of bladder cancer has been described in several studies [7,14,16-18].

A strong inverse dose response between water intake and bladder cancer was found in women, $\mathrm{OR}=0.3$ [95\% CI: 0.1-0.8]. The public water used by the participants in the study by Wilkens et al. [7] was ground water, filtered through volcano rocks and probably free of contaminants. Male cases drank more fluid than controls; female cases drank much less than controls. However the protective effect of tap (ground) water was stronger in females, especially in female smokers. It is evident that chemical carcinogens in cigarette smoke contribute markedly to the pathogenesis of bladder cancer. The relative effect of high water intake, as compared with smoking is highlighted in this study [7]. The strongest effect was for tap water. When limited to transitional cell (urothelial) carcinoma only (88\%), the results were similar [7].

High fluid intake reduces probably the exposure of the bladder urothelium to carcinogens by diluting the urine and causing more frequent voiding. The carcinogenic potential of chlorinated byproducts in public water was evaluated. Michaud et al. [16] carried out a prospective study on 47,909 men and showed also that the total daily fluid intake was inversely related to the risk of bladder cancer, $\mathrm{OR}=0.51$ [95\% CI: $0.32-0.80]$ for $>2,531 \mathrm{ml} / \mathrm{d}$ versus $<1,290 \mathrm{ml} / \mathrm{d}$. The results were more significant for water intake, OR $=0.49$ [95\% CI: $0.28-0.86$ ] for $>1,440 \mathrm{ml} / \mathrm{d}$ versus $<240 \mathrm{ml} / \mathrm{d}$ [16]. Seventy five percent of cases of bladder cancer arise in individuals who drink public water. According to the above authors, evaluating the exposure of these patients to chlorinated byproducts is a complex task. Despite the obscure role of chlorinated products, findings suggest that in the US, high water consumption may reduce the risk of bladder cancer by about $50 \%$ $[16,19]$. Michaud et al. [17] found a 39\% decrease in bladder cancer risk for both genders, between the highest and lowest level of total fluid intake (statistically significant). Exclusion of trihalomethane (THM - 
chlorination byproducts) levels from the model did not alter the results. With regard to water intake, there was a $53 \%$ decrease in risk for males and females consuming $>1,400 \mathrm{ml} / \mathrm{d}$ when compared with $<400 \mathrm{ml} / \mathrm{d}$.

It is not clear why beverages other than water are not inversely associated with bladder cancer risk. Inconsistencies, as demonstrated in numerous studies, may be caused by different degrees of exposure to contaminants, which may vary from one population to the next, and may be due in part to different methodologies used. In contrast to the results reported by Villanueva [8], who showed that an increase of 1 $1 / \mathrm{d}$ in fluid intake may lead to up to an $8 \%$ increase in bladder cancer risk in men, in Michaud et al. study[17], higher tap water consumption reduced the risk of bladder cancer, even in the presence of the highest THM levels. Fluid intake during the years close to diagnosis seems to be irrelevant, given the long latency of bladder cancer [17].

Higher water intake was associated with a slight decrease in bladder cancer with greater protection for women ( $\mathrm{p}$ for trend, .039). Of note, this association was also modified by daytime voiding frequency (inverse association if $\geq 6 \mathrm{X}$ voiding /d). Jiang.s study [14] suggested a slight reduction in bladder cancer risk with water intake. Nocturia in women was related to an increased risk of bladder cancer. In women, nocturia has been linked with lesions like cystitis glandularis and malignancy, while in men it is related to benign prostatic hyperplasia [14].

Experimental studies have suggested that increased voiding frequency may reduce bladder cancer if carcinogens are present in the urine. The two human studies on this issue are limited and controversial. A large multicentre case-control study has shown an inverse risk of bladder cancer with increasing nocturia in both men and women, with a reduction of $40-50 \%$ in bladder cancer risk. The protective effect was related to the amount of water absorbed. It seems that the duration of contact of carcinogens with the urothelium may be shortened in the presence of nocturia. Increased micturition frequency together with possible urine dilution due to increased water consumption may reduce the effect of carcinogens on the urothelium [18].

\section{Dehydration and Direct Association with Bladder Cancer}

The difficulty in evaluating fluid consumption in humans has been described. Fluid intake has been variously associated with a moderate increase in bladder cancer risk, decreased risk, and no effect. A decrease in fluid consumption can lead to a high concentration of carcinogens or to prolonged contact between carcinogens and the urothelium [20].

Others [21] found that total fluid consumption is associated with a high risk of bladder cancer in men. Consumption of more than 3.5 $1 / \mathrm{d}$ of fluid carried a 1.33 risk of bladder cancer as compared with $<2$ $1 / \mathrm{d}$. There was no increase in risk in women. Two $1 / \mathrm{d}$ versus $0.5 \mathrm{l} / \mathrm{d}$ of tap water carried a risk of bladder cancer, OR=1.46 [95\% CI: 1.20 1.78]. This increased risk of cancer suggests that a carcinogenetic chemical in tap water is active in the malignant transformation (coffee or chlorinated byproducts). Since water is a universal solvent, it is practically impossible to evaluate the isolated fluid effect. The highly variable results obtained from the numerous studies may be explained by the heterogeneity of the water components [21]. Villanueva [8] suggested that the fluid itself is probably not connected with the risk of bladder cancer. In a previous study, a public water system with chlorination was found to increase the risk of bladder cancer by 1.4 [21].
Brinkman and Zeegers [22] found no relationship between total fluid intake and bladder cancer risk. They found that most studies reviewed in their paper on this issue were case-control studies. A small majority of studies $(6 / 10)$ reporting a statistically significant association were in favor of a positive link between fluid intake and the risk of bladder cancer, mainly among men.

Hemelt et al. [23] found that increased fluid consumption was associated with a significantly decreased risk of bladder cancer, OR, 0.93 [95\% CI, 0.88-0.99] per cup in men and in both genders combined. Water consumption was not significantly related, although water was the main fluid consumed.

More recently, a cohort study on the relation between total fluid intake and risk of urothelial cell carcinoma (UCC) found no association with water consumption, HR, 1.12 [95\% CI, 0.86-1.45] p-trend $=0.42$. Normal total fluid intake is not associated with UCC risk [24].

\section{Colorectal Cancer, Constipation and Fluid intake}

A strikingly significant inverse association between the number of glasses of tap water $(>5 / \mathrm{d}$ versus $\leq 2 / \mathrm{d})$ and risk of colorectal cancer (CRC) has been observed in women, OR, 0.55 [95\% CI: 0.31-0.99], $\mathrm{p}$ for trend 0.004 . This suggests that higher water consumption may reduce CRC risk by decreasing the colon transit time or by decreasing the carcinogen concentration in the colon water phase (the amount of water lost daily in the feces) [24].

Fifteen symptoms of colorectal cancer (CRC) were recorded in 194 patients: $51 \%$ presented with changes in bowel habits that included constipation in 53 (27\%) that lasted 3-20 weeks (mean 10 weeks); altered stools in 48 (25\%) of 9 weeks mean duration with stool impaction. No association was noted between duration of symptoms and CRC stage. These symptoms are also found in the general population but with different intensity. No mention is made in Majundar article [25] on fluid intake and its role in constipation or CRC. Constipation is an independent predictor, OR=3.16 [95\% CI: 1.38-7.24]. In this study, constipation appeared to be one of the symptoms of CRC, but was not considered to play a pathogenic role [25].

Colorectal cancer is considered the second most common cancer in women and the third most common cancer in men. Of note, although water intake is important for digestion and gut function, it is rarely measured [20]. The latter author also mentioned that 80-200 $\mathrm{ml}$ of water is lost daily through feces. With reduced intake this fluid content may diminish, which may lead to constipation. In animals this may cause an increased tendency to neoplasms. Increased water consumption may decrease colon transit time and by reducing contact between carcinogens and the bowel mucosa, may decrease the risk of CRC. In men a high water intake was protective for distal CRC, OR, 0.68 [95\% CI, 0.49-0.96]. No such finding was evident among women [26]. But a study from Taiwan described an inverse relation between water intake and CRC in men, which was particularly strong for rectal cancer [27].

Mild dehydration has been evaluated as a risk factor for constipation. The term constipation is defined in various ways, which may cause confusion: change in frequency, volume or consistency with a sensation of incomplete evacuation. Fiber, carbohydrate and fluid intake may play a role. As far as fluid consumption is concerned, it is relevant only in the context of hypohydration and dehydration. Fluid 
intake is still mentioned with regard to constipation, but only out of tradition, as it has never been proven. This may be due to the lack of a simple test to evaluate dehydration [28].

Muller-Lissner, et al. [29] studied myths and misconceptions about chronic constipation. They concluded that: "There is no evidence that constipation can successfully be treated by increasing fluid intake, unless there is evidence of dehydration".

An attempt to evaluate the etiology of chronic constipation was made by Leung [30]. Thirty-six elderly men were followed, 18 with constipation. Patients with constipation ate fewer meals and fewer calories, but the same amount of fiber. Slow colonic transit was related to low caloric intake $(\mathrm{p}<0.0001)$ and a low fluid intake $(\mathrm{p}<0.05)$. The author mentioned that very little is known about the etiology of chronic constipation and that all published material is based on legend. Accordingly, a well-designed study is required.

Total fluid intake has been suggested to protect against CRC [31]. The authors examined the association in a cohort of 10,852 patients. After 13.3 years, 1,443 males and 1,040 female patients were diagnosed with CRC. A non-significant positive trend for total fluid intake was found for rectal cancer $(\mathrm{p}=0.08)$. The authors concluded that total fluid intake was not associated with CRC risk in men or women.

\section{Conclusions}

The many limitations in our ability to evaluate mild dehydration, the subtle differences between low water intake and mild dehydration, and the complex pathogenesis of the diseases described, all prevent the clear-cut identification of hydration as a major factor in the prevention and treatment of these diseases.

Tabulation of results was not adopted because of the widely variable definitions, methods and nature of fluids used in the different articles compared.

High water intake for the prevention and treatment of UTIs may not be a legend after all, as it has repeatedly been shown to be effective in dehydrated patients. The subsequent water diuresis that flushes the urinary tract with more frequent micturition should prevent UTI or assist in the cure of the disease.

Several studies have presented epidemiological evidence of an increased risk of bladder cancer with increased fluid or water consumption or no association thereof. However, a large minority of studies points to a reduced risk of bladder cancer as water intake increases. Inconsistencies in the findings may be due to differences in the types of beverages and methods involved the inability to evaluate the volume of fluids absorbed, and the possible differences in the type and content of carcinogens in the tap water of the different studies. In addition, some studies were of a case-control nature, whereas others were cohort studies. Of note is the conclusion of one study that although the increased risk of bladder cancer was probably carcinogen-related, contaminant-free water does not play a role in the association between high water consumption and increased bladder cancer risk [8].

With adequate hydration the colon transit time is shortened, which, in addition to a normal colonic water phase, should prevent prolonged contact of carcinogens with the colonic mucosa and the development of colorectal cancer.

\section{Acknowledgements}

This study was supported in part by Kibbutz Sde-Boker, Israel.
Thanks are given for their thorough review of the manuscript to the members of the Department of Drylands Ecology, at the Midreshet Ben-Gurion Campus of the Ben Gurion University of the Negev.

\section{Con}

No conflict of interest is declared.

\section{References}

1. Sawka MN, Cheuvront SN, Carter R 3rd (2005) Human water needs. Nutr Rev 63: S30-S39.

2. Popkin BM, D’Anci KE, Rosenberg IH (2010) Water, hydration, and health. Nutr Rev 68: 439-458.

3. Ferry M (2005) Strategies for ensuring good hydration in the elderly. Nutr Rev 63: S22-S29.

4. Manz F, Wentz A (2005) The importance of good hydration for the prevention of chronic diseases. Nutr Rev 63: S2-S5.

5. Manz F (2007) Hydration and disease. J Am Coll Nutr 26: 535S-541S.

6. Kunze E, Chang-Claude J, Frentzel-Beyme R (1992) Life style and occupational risk factors for bladder cancer in Germany: A case control study. Cancer 69 : 1776-1790.

7. Wilkens LR, Kadir MM, Kolonel LN, Nomura AM, Hankin JH (1996) Risk factors for lower urinary tract cancer: the role of total fluid consumption, nitrite and nitrosamines and selected foods. Cancer Epidemiol Biomarkers Prev 5: 161166.

8. Villanueva CM, Cantor KP, King WD, Jaakkola JJ, Cordier S, et al. (2006) Total and specific fluid consumption as determinant of bladder cancer risk. Int J Cancer 118: 2040-2047.

9. Shannon J, White E, Shattuck AL, Potter JD (1996) Relationship of food groups and water intake to colon cancer risk. Cancer Epidemiol Biomarkers Prev 5 : 495-502.

10. Beetz R (2003) Mild dhydration: a risk factor of urinary tract infection? Eur $J$ Clin Nutr 57: S52-S58.

11. Stauffer CM, van der Weg B, Donadini R, Ramelli GP, Marchand S, et al. (2004) Family history and behavioral abnormalities in girls with recurrent urinary tract infection: a cotrolled study. J Urol 171:1663-1665.

12. Rudaitis S, Pundziene B, Jievaltas M, Uktaveris R, Kevelaitis E (2009) Recurrent urinary tract infection in girls: do urodynamic, behavioral and functional abnormalities play a role? J Nephrol 22: 766-773.

13. Elstad EA, Maserejian NN, McKinlay JB, Tennstedt SL (2010) Fluid manipulation among individuals with lower urinary tract symptoms: a mixed method study. $J$ Clin Nurs 20: 156-165

14. Jiang X, Castelao JE, Groshen S, Cortessis VK, Shibata D, et al. (2009) Urinary tract infection and reduced risk of bladder cancer in Los Angeles. $\mathrm{Br} \mathrm{J}$ Cancer 100: 834-839.

15. Oyasu R, Hopp ML (1974) The etiology of cancer of the bladder. Surg Gynecol Obstet 138: 97-108.

16. Michaud DS, Spiegelman D, Clinton SK, Rimm EB, Curhan GC, et al. (1999) Fluid intake and the risk of bladder cancer in men. N Engl J Med 340: 13901397.

17. Michaud DS, Kogevinas M, Cantor KP, Villanueva CM, Garcia-Closas M, et al. (2007) Total fluid and water consumption and the joint effect of exposure to disinfection by-products on risk of bladder cancer. Environ Health Perspect 115: 1569-1572.

18. Silverman DT, Alguacil J, Rothman N, Real FX, Garcia-Closas M, et al. (2008) Does increased urination frequency protects against bladder cancer? Int $J$ Cancer 123: 1644-1648.

19. Bar David Y, Gesundheit B, Urkin J, Kapelushnik J (2004) Water intake and cancer prevention. J Clin Oncol 22: 383-385

20. Altieri A, La Vecchia C, Negri E (2003) Fluid intake and risk of bladder and other cancers. Eur J Clin Nutr 57: S59-S68. 
Citation: Benharroch D, Ariad S (2012) Mild Dehydration - Possible Association with Bladder and Colorectal Cancers - A Review. J Food Process Technol 3:142. doi:10.4172/2157-7110.1000142

Page 5 of 5

21. Villanueva CM, Fernandez F, Malats N, Grimalt JD, Kogevinas M (2003) Metaanalysis of studies on individual consumption of chlorinated drinking water and bladder cancer. J Epidemiol Community Health 57: 166-173.

22. Brinkman M, Zeegers MP (2008) Nutrition, total fluid and bladder cancer. Scand J Urol Nephrol 42: 25-36

23. Hemelt M, Hu Z, Zhong Z, Xie LP, Wong YC, et al. (2009) Fluid intake and the risk of bladder cancer: results from South and East China case-control study on bladder cancer. Int J Cancer 127: 638-645.

24. Ros MM, Bas Bueno-de-Mesquita HB, Büchner FL, Aben KK, Kampman E, et al. (2011) Fluid intake and risk of urothelial cell carcinomas in the European Perspective Investigation into Cancer and Nutrition (EPIC). Int J Cancer 128: 2695-2708.

25. Majumdar SR, Fletcher RH, Evans AT (1999) How does colorectal cancer presents? Symptoms duration and clues to location. Am J Gastroenterol 94: 3039-3045.

26. Slattery ML, Caan BJ, Anderson KE, Potter JD (1999) Intake of fluids and methylxantine-containing beverages: association with colon cancers. Int $\mathrm{J}$ Cancer 81: 199-204.

27. Tang R, Wang JY, Lo SK, Hsieh LL (1999) Physical activity, water intake and risk of colorectal cancer in Taiwan: a hospital-based case-control study. Int J Cancer 82: 484-489.

28. Arnaud MJ (2003) Mild dehydration: a risk factor of constipation? Eur J Clin Nutr 57: S88-S95.

29. Mueller-Lissner SA, Kamm MA, Scarpinato C, Wald A (2005) Myths and misconceptions about chronic constipation. Am J Gastroenterol 100: 232-242.

30. Leung FW (2007) Etiologic factors of chronic constipation: review of the scientific evidence. Dig Dis Sci 52: 313-316.

31. Simons CC, Leurs LJ, Weijenberg MP, Schouten LJ, Goldbohm RA, et al. (2010) Fluid intake and colorectal cancer risk in the Netherland Cohort Study. Nutr Cancer 62: 307-321. 